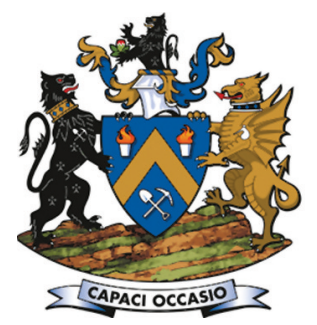

\title{
The effect of geological uncertainty on achieving short-term targets: a quantitative approach using stochastic process simulation
}

\author{
by M. Soleymani Shishvan* and J. Benndorf*
}

\section{Synopsis}

Continuous mining systems containing multiple excavators producing multiple products of raw materials are highly complex, exhibiting strong interdependency between constituents. Furthermore, random variables govern the system, which causes uncertainty in the supply of raw materials: uncertainty in knowledge about the reserve, the quantity demanded by the customers, and the breakdown of equipment. This paper presents a stochastic-based mine process simulator capturing different sources of uncertainties. It aims to quantify the effect of geological uncertainty and its impacts on the ability to deliver contractually defined quantities and qualities of coal, and on the system efficiency in terms of utilization of major equipment. Two different areas of research are combined: geostatistical simulation for capturing geological uncertainty, and stochastic process simulation to predict the performance and reliability of a large continuous mining system.

The process of modelling and simulation in this specific production environment is discussed in detail. Problem specification and a new integrated simulation approach are presented. A case study in a large coal mine is used to demonstrate the impacts and evaluate the results in terms of reaching optimal production control decisions to increase average equipment utilization and control coal quality and quantity. The new approach is expected to lead to more robust decisions, improved efficiencies, and better coal quality management.

\section{Keywords}

continuous mining, scheduling, stochastic process simulation, geological uncertainty. indicators of a complex system. In the past few years there has been significant development in the applications of process simulation in the mining industry. Panagiotou (1983) described the application of the simulation program SIMPTOL for opencast lignite mines that use bucket wheel excavators (BWEs), conveyors, and stackers. The main objective was to select and match the equipment to fit material characteristics while meeting production requirements and mine profiles.

Michalakopoulos et al. (2005) presented a simulation model of an excavation system at a multi-level terrace mine using the GPSS/H simulation language. The principal model output variables are production and arrival rate of product and waste at the transfer point. Michalakopoulos et al. (2015) utilized Arena simulation software for the simulation of the Kardia Field mine in Greece. Validation of the results illustrates an acceptable agreement with the actual data. Fioroni et al. (2007) used discrete tools for simulation of continuous behaviour for modelling the conveyor belt network of a large steelmaking company. The authors proposed a modelling approach to the flow process that uses portions of materials and treats them as discrete entities in simulation modelling. The results demonstrated that this technique was valid and successful. Salama et al. (2014) used a combination of discrete event simulation and mixed integer programming (MIP) as a tool to improve decision-making in underground mining. The proposed method uses the simulation approach to evaluate the operating costs of different haulage system scenarios and obtains the cash flows for input into the MIP model.

* Department of Geoscience and Engineering, Delft University of Technology, Delft, Netherlands.

(c) The Southern African Institute of Mining and Metallurgy, 2016. ISSN 2225-6253. This paper was first presented at the, 23rd International Symposium on Mine Planning and Equipment Selection (MPES) 2015, 8-11 November 2015, Sandton Convention Centre, Johannesburg, South Africa. 


\section{The effect of geological uncertainty on achieving short-term targets}

The reviewed literature demonstrates that the stochastic process simulation is a potent method for measuring the key performance indicators (KPIs) in continuous mining systems. However, the investigation of the impacts of geological uncertainty in the performance of continuous mining systems is still seen as a major gap. This paper presents a stochasticbased mine process simulator focusing on the effects of geological uncertainty to predict the mine process performance and reliability. The following section specifies the problem of stochastic simulation of continuous mining systems. Thereafter, the procedure for a new integrated simulation approach is discussed. In this study, a discretecontinuous methodology is proposed and Arena simulation software (Rockwell Automation Technologies Inc. 2012) is used for modelling. As a case study, a completely known data-set is analysed and the results presented.

\section{Problem description}

In general, continuous coal mining systems contain parallel production lines that begin with excavators, followed by material transport by conveyor belts and distribution at the mass distribution centre where material is divided into two destinations, namely, the coal bunker and the waste materials dump. Waste materials are dumped by spreaders at the dump while lignite is stacked by the stacker in the stockpile yard. The reclaimer and a network of conveyor belts are used for loading lignite into railway wagons. Finally, the product is despatched to customers (mostly power plants) based on their daily demands.

The problem considered here is to quantify the effect of geological uncertainty and its impact on the ability to deliver contractually defined coal quantities and qualities, and on the system efficiency in terms of utilization of major equipment. The KPIs of the system are defined as the ability to meet coal quality and quantity targets and the utilization of the system. The parameters of the KPIs to be evaluated for each simulation replication are presented in Table I. More details about the mathematical formulation of evaluation function and parameters are discussed in Shishvan and Benndorf (2014).

As an example, Figure 1 shows a scatter plot that illustrates the relationship between two ash contents measured on the same samples. It appears from this figure that as the variable on the vertical scale (ash content measured in the laboratory) changes, the variable on the horizontal scale (ash content based on the estimated model) seems to vary randomly within a relatively small range without tending to increase or decrease significantly. It can be seen that the reality (laboratory measurements) shows a significant higher fluctuation compared to the estimated model. There is a weak relationship between two variables, with a correlation coefficient of 0.182 in the scatter plot. These observations give rise the question 'where do these fluctuations come from?' which is investigated in this paper.

\section{Integrated simulation approach}

When using interpolated reserve models as a basis for mine planning, a smoothing effect is to be expected. This is due to the nature of spatial interpolators, which are often designed to minimize the estimation errors. Alternatively, conditional simulation methods in geostatistics have been developed to quantify variability and uncertainty associated with the geology (Chiles and Delfiner, 2012). These techniques result in a set of equally probable scenarios defining the spatial distribution of attributes within a deposit (realizations), which capture in-situ variability as found in the data. Local differences between these realizations can be used for mapping uncertainty. The applicability to multi-seam coal deposits was demonstrated by Benndorf (2013a, 2013b).

On the other hand, the process simulation model of the continuous mining system is intended to reproduce the operational behaviour in a real opencast coal mine. The extraction and conveying of lignite and waste are emulated in a combined discrete-continuous stochastic environment. This allows incorporation of uncertainty associated with the geological block model. It allows the re-creation of the

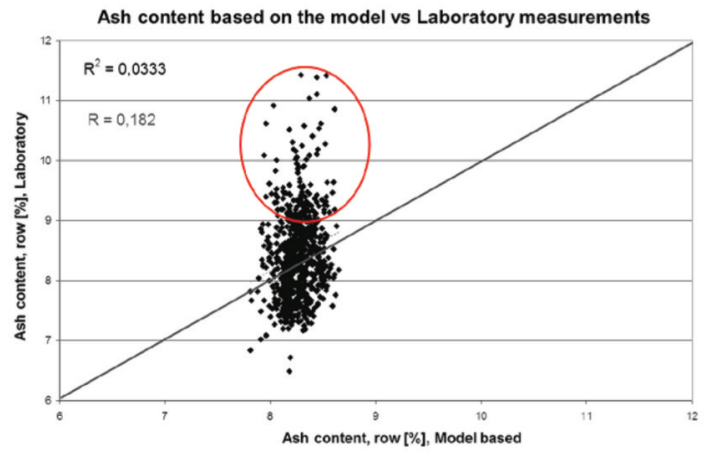

Figure 1-Scatter plot of ash content of product delivered to the power plants

\section{Table I}

\section{The parameters of evaluation function of the KPls}

\begin{tabular}{|l|l|}
\hline Objective & Evaluation of aggregated KPIs of each simulation replication based on the pre-defined short-term planning targets \\
\hline KPIs & $\begin{array}{l}\cdot J_{1} \text { - coal quality: should be between defined lower and upper limits, otherwise penalties should be applied } \\
\cdot J_{2}-\text { coal quantity: should be between defined lower and upper limits, otherwise penalties should be applied } \\
\cdot J_{3} \text { - utilization: average utilization of the system can be derived from the average utilization of excavators }\end{array}$ \\
\hline Decision variables & $\begin{array}{l}\text { - Task schedules: different alternatives for short-term plans (daily/weekly/monthly) } \\
\text { - Extraction sequences: sequence of extracting mining blocks for each excavator } \\
\text { - Extraction rate of excavators in the different time spans }\end{array}$ \\
\hline Constraints & variables management: the quality and the quantity of coal needed in the stock yard influences other decision \\
& $\begin{array}{l}\text { - Each block can be mined just once } \\
\text { - The conveyor belt can be moved further along only if all the blocks in one pass are mined out }\end{array}$ \\
\hline
\end{tabular}




\section{The effect of geological uncertainty on achieving short-term targets}

deterministic and/or random occurrences of events such as operating stoppages caused by unavailability of spreaders or conveyor belts, equipment failures, and preventive and corrective maintenance activities.

Dowd and Dare-Bryan (2005) explored the general concepts of the integration of the geostatistical simulation within the entire design and production cycle. The authors illustrated these concepts with particular reference to blast modelling. This paper aims to combine the two simulation concepts, namely geostatistical simulation for capturing geological uncertainty and stochastic process simulation, to predict the performance and reliability of a large continuous mining system. Figure 2 shows the integrated simulation approach.

In this approach, realizations based on conditional simulation and an interpolated model using kriging are considered as input for the mine process simulator. The kriged model is used for comparison. The software selected to implement the integrated simulation approach is Rockwell ARENA 14.5, which permits close reproduction of the behaviour of complex real systems with complicated decision logic. The software offers intuitive flowcharting support to the modelling, control over the flow of entities in the system, custom statistics, user-defined expressions, and interfacing with external databases and spreadsheets (Kelton and Law 2000). The output of the simulator is the set of values for each KPI. At this stage, penalties are applied when deviating from production targets. The KPIs are summarized in an evaluation function, which results in a probability distribution when multiple replications are evaluated (Figure 2).

\section{Case study and implementation aspects}

\section{System description}

The objective of this study is to illustrate the effect of geological uncertainty on the performance of a complex continuous mining system. To analyse the performance of the proposed approach, the case study is presented in a completely known and fully controllable environment. In this regard, the Walker Lake data-set as a completely known environment is chosen (Isaaks and Srivastava 1989). The real value block model (complete Walker Lake data-set), an average-type estimated block model using ordinary kriging, and 20 conditionally simulated realizations using sequential Gaussian simulation of the deposit are used as different replications for building the simulation experiments.
Figure 3 schematically shows a typical opencast mine. The mining operation uses six BWEs at six benches. Extracted material is transported by conveyor belts to the mass distribution centre. Here, destinations are determined based on the type of materials and the excavator from which they were derived. Finally, waste materials are conveyed to spreader no. 1 or 2 and the coal to the stockpile yard.

The block model is divided into six equal areas and each area is assigned to one excavator (Figure 4).

The major steps in simulation modelling are as follows:

- The first step is to define appropriate entities. Entities are the block portions to be extracted in each period

> The second step is to assign block attributes. As an entity enters the system its attributes, consisting of block coordinates ( $\mathrm{x}, \mathrm{y}$, and $\mathrm{z}$ ), block tonnage, block

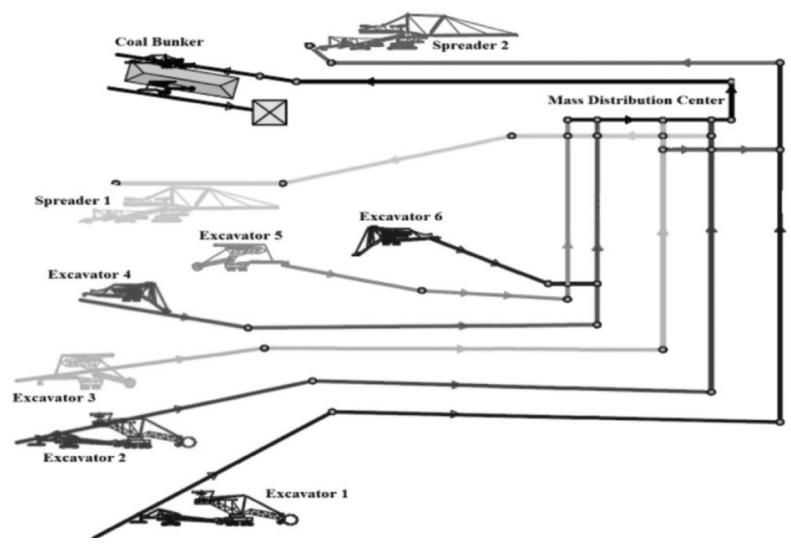

Figure 3-Schematic view of the problem (opencast mine)

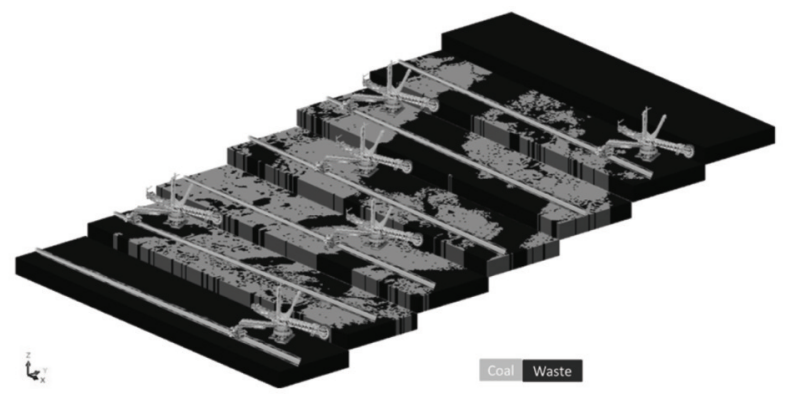

Figure 4-Block model, assigned area for the excavators

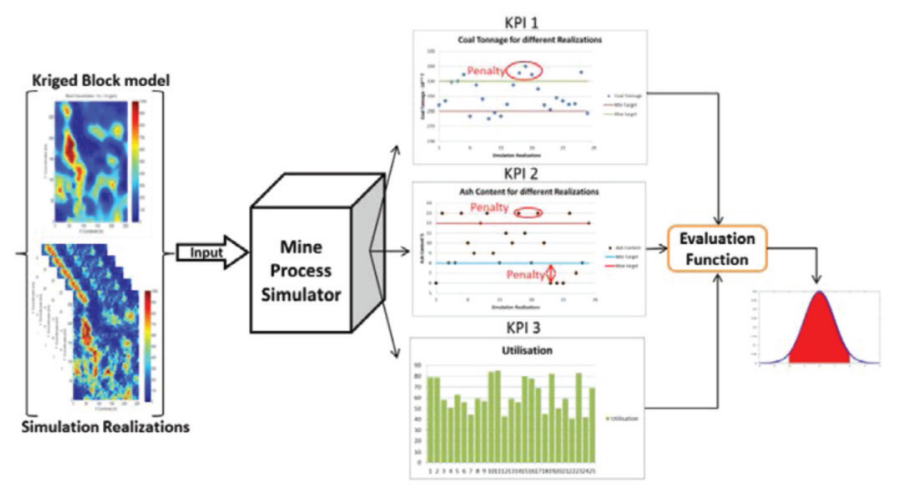




\section{The effect of geological uncertainty on achieving short-term targets}

type, quality parameters, and destination, are assigned. These attributes are read from the geological block model

> Subsequently, the entity is placed in a queue for extraction by the excavator as a resource module

$>$ Each entity has a delay based on operating time, after which it is released

> At the final step, variables such as total waste tonnage and ore tonnage entering the system are calculated.

A capacity constraint is implemented to prevent overflow of loose material on the conveyor belt that is connected to the coal bunker. Based on the maximum amount of coal that can be on the belt, a constraint of $6000 \mathrm{~m}^{3} / \mathrm{h}$ is considered for coal. When the production rate exceeds these limits, the model starts to identify the excavators that are producing coal. The excavator that corresponds to the minimum production rate is set to standby.

Decision variables of this case consist of:

> Task schedule-working schedule for a time horizon of 7 days is given in Table II. This mine operates 24 hours per day in three working shifts. As an example, in Table II, the number 110 shows that the corresponding equipment is available for first and second shifts and is not available for the third shift

> Extraction sequence-in this case, considered to be a constant (from one side of bench to the other side) without any movement of excavators during the excavation

- Extraction rate of excavators -in this case, assumed to be equal to the theoretical capacity of excavators (Table III)

> Stockpile management-in this case, if the stockpile for a specific coal type is full, the excavator(s) that produces that type of coal should be idled until stockpile space is available.

Tables III summarize the general information and technical parameters that are used for the simulation model building.

\section{Results and discussion}

The results of 22 different block models (simulation replications, $r$ ): real, estimated, and 20 realizations are analysed in the specified time horizon (one week, in this case, i.e., 168 hours). The total extracted coal tonnages for the different scenarios are presented in Figure 5. The system simulation based on the estimated model shows significantly less coal (10\%) than the average of the 20 realizations. On the other hand, the real model shows a very similar value to the average value of the 20 realizations. Clearly, the estimated model underestimates coal production for the defined schedule. This is due mainly to ignoring in situ variability and geological uncertainty. The capability of conditional simulation to quantify geological uncertainty improves the prediction of system performance.

Note that the application of average-type estimated models does not always lead to underestimation. Depending on local geological conditions, these techniques may also lead to an overestimation.

Figure 6 presents the average ash content of extracted coal for the real model (light grey), the estimated model (black), the realizations (dark grey), and the average of simulation (red line). The ash contents of all realizations substantially exceed the value predicted by the estimated deposit model. The average is again similar to the real value. Relying on the estimated model would indicate a biased and over-optimistic ash content.

In this study three KPIs are measured and penalties are applied for not meeting the coal quality target (Figure $7 \mathrm{a}$ ), quantity targets (Figure $7 \mathrm{~b}$ ), and equipment utilization target (Figure 8a). The values in Figure 7 are calculated based on parameters specified in Table IV. The costs of deviation from the targets (the penalties) in this study are one unit for one ton of coal. Hence, these penalties can be interpreted as percentage and tonnage of deviation from the targets.

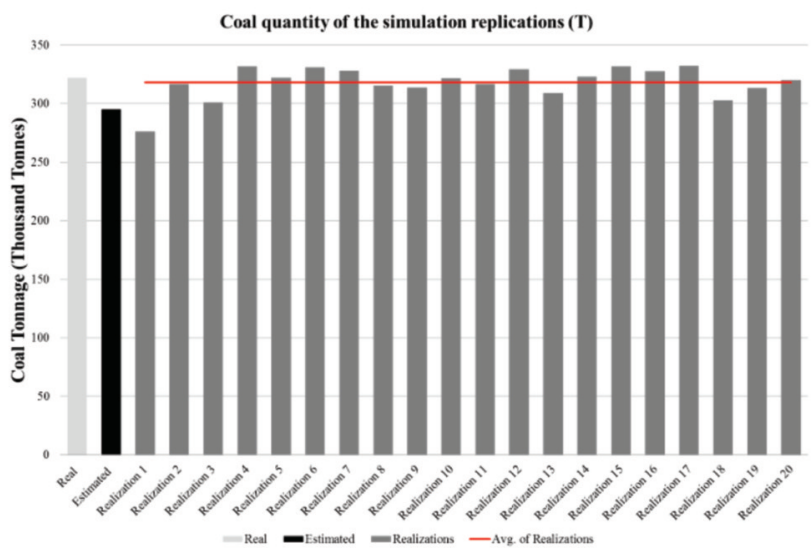

Figure 5-Illustrative results of different scenarios, coal tonnage

\begin{tabular}{|c|c|c|c|c|c|c|c|}
\hline \multicolumn{8}{|c|}{$\begin{array}{l}\text { Table /I } \\
\text { Working schedule of the equipment as a decision } \\
\text { variable }\end{array}$} \\
\hline \multirow{2}{*}{$\begin{array}{l}\text { Type of } \\
\text { equipment }\end{array}$} & \multicolumn{7}{|c|}{ Days } \\
\hline & 1 & 2 & 3 & 4 & 5 & 6 & 7 \\
\hline Excavator 1 & 111 & 001 & 111 & 111 & 111 & 111 & 111 \\
\hline Excav & 111 & 111 & 111 & 001 & 111 & 111 & 111 \\
\hline Excavator 3 & 111 & 011 & 111 & 001 & 111 & 111 & 111 \\
\hline Excavator 4 & 111 & 111 & 111 & 111 & 111 & 111 & 111 \\
\hline Exc & 111 & 111 & 001 & 111 & 111 & 111 & 111 \\
\hline Exc & 111 & 110 & 110 & 111 & 111 & 111 & 111 \\
\hline Spreac & 111 & 111 & 111 & 001 & 111 & 111 & 111 \\
\hline Spread & 111 & 001 & 111 & 111 & 111 & 111 & 111 \\
\hline Conveyor belts & 111 & 111 & 111 & 111 & 111 & 111 & 111 \\
\hline
\end{tabular}

Table III

General information on equipment

\begin{tabular}{|l|c|c|}
\hline Type & $\begin{array}{c}\text { Theoretical capacity } \\
\text { (loose, } \mathbf{~ m}^{\mathbf{3} / \mathbf{h})}\end{array}$ & $\begin{array}{c}\text { Scheduled time } \\
\boldsymbol{T}_{\text {scheduled }} \text { (h) }\end{array}$ \\
\hline Excavator 1 & 4900 & 152 \\
Excavator 2 & 4900 & 152 \\
Excavator 3 & 3770 & 144 \\
Excavator 4 & 1400 & 168 \\
Excavator 5 & 3770 & 152 \\
Excavator 6 & 740 & 152 \\
Spreader 1 & 10000 & 152 \\
Spreader 2 & 10000 & 152 \\
Conveyor belts & 6000 & 168 \\
\hline
\end{tabular}




\section{The effect of geological uncertainty on achieving short-term targets}

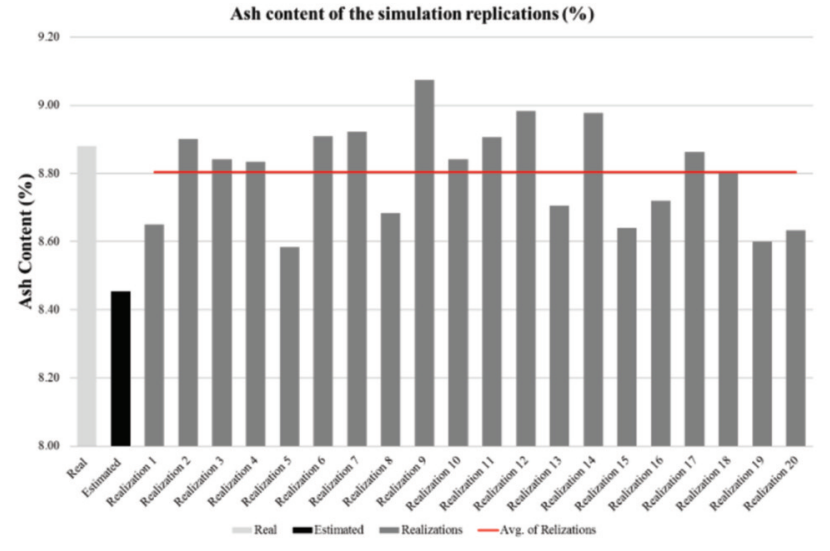

Figure 6-Illustrative results of different scenarios, ash content

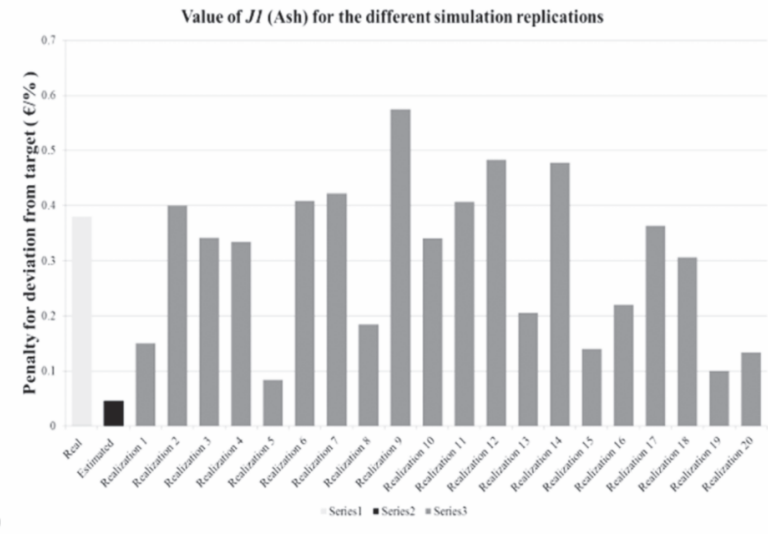

(a)

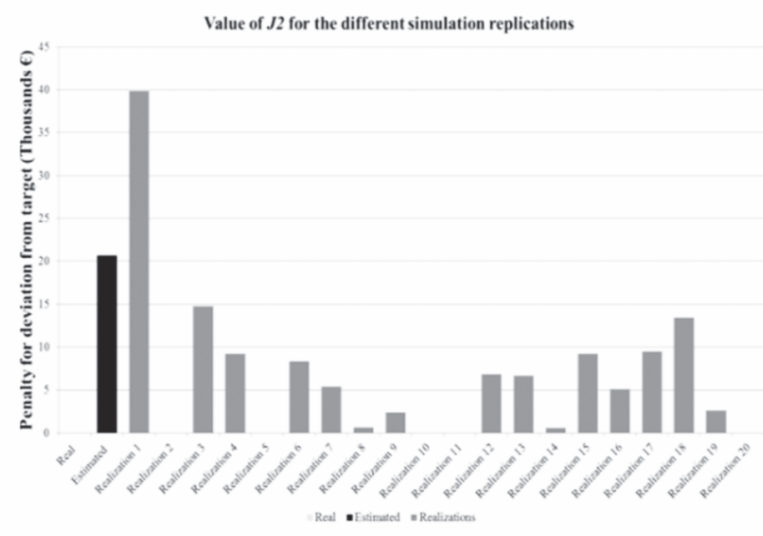

(b)

Figure 7-(a) Coal quality KPI, (b) coal quantity KPI

Figure $7 \mathrm{~b}$ also indicates that, for example, realization 1 will lead to an underproduction of $40 \mathrm{kt}$ of coal. To account for this uncertainty the stockpile inventory should be at least $40 \mathrm{kt}$ before the start of the week to accommodate potential deviations from target and secure a sufficient supply to customers. Figure 8 demonstrates the average utilization of the system for different realizations and shows box plots of the utilization of each excavator. Evidently, geological uncertainty and variability have a significant impact on the measured KPIs.
Figure 9 shows the ash content of a week's production to be delivered by rail to the power plants. The results reveal that predictions based on the estimated model (black line) and the reality (dark grey line) are not well correlated. This means that the prediction based on an interpolated model has limits. When considering the conditional simulation model, there are 20 realizations (light grey cloud) and the average of the realizations (red line), which is the stochastic prediction. Comparing these with the reality, the red line generally follows the true ash content very well. Deviations are in the expected range of deviations, which are mapped by the shadow range (realization cloud).

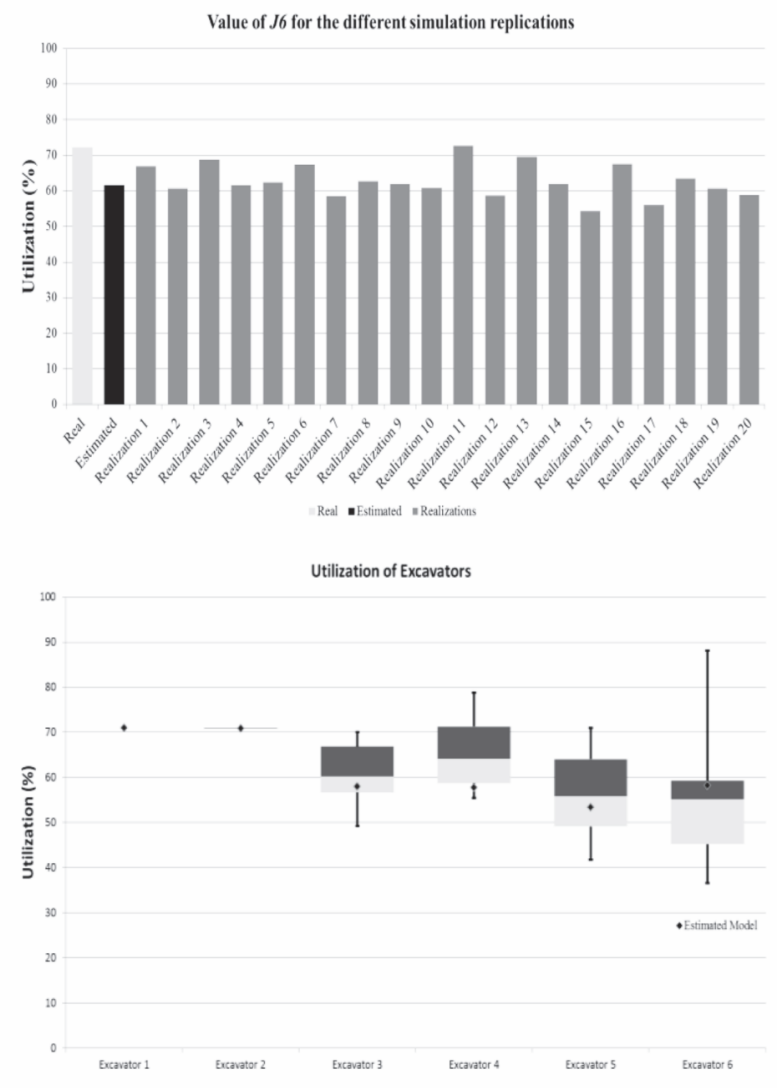

Figure 8-(a) Utilization KPI, (b) box plots of utilization of excavators

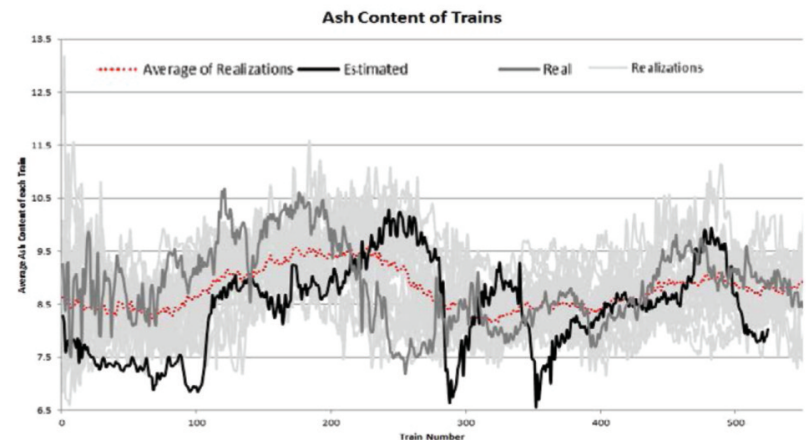

Figure 9-Average ash content in a week's production 


\section{The effect of geological uncertainty on achieving short-term targets}

Previous examples illustrated that stochastic system simulation is a valid and powerful tool for exploring the effect of geological uncertainty on the expected performance of complex continuous mining systems. It provides the mine planning engineer with a valuable tool to foresee critical situations affecting the continuous supply of raw material to customers and the system performance.

\section{Conclusions}

Continuous mining systems require large investments and have high operational costs. Decisions in daily scheduling are impacted by uncertainties such as incomplete knowledge about the deposit, which can have a significant impact of actual production performance. This contribution has proposed a simulation-based framework where the method of geostatistical simulation has been integrated with mine system simulation to account for the effects of geological uncertainty. Results show that such an approach provides the mine planning engineer with a valuable tool to foresee critical situations affecting the continuous supply of raw material to customers and the system performance.

Future research will be carried out to extend the system simulation to also to capture stochastic downtime behaviours and stochastic demand. For optimal control decisions, the simulation approach will be integrated to a simulation-based framework for optimization of short-term mine planning and operations control (Benndorf, 2014; Benndorf et al., 2014).

\section{References}

BENNDORF, J. 2013a. Application of efficient methods of conditional simulation for optimising coal blending strategies in large continuous open pit mining operations. International Journal of Coal Geology, vol. 112. pp. 141-153.

BENNDORF, J. 2013b. Investigating in situ variability and homogenisation of key quality parameters in continuous mining operations. Mining Technology, vol. 122 , no. 2. pp. $78-85$.

BENNDORF, J. 2014. Moving towards real-time management of mineral reserves - a geostatistical and mine optimization closed-loop framework. Mine Planning and Equipment Selection. Springer International. pp. 989-999.

Benndorf, J., Buxton, M.W.N., and Shishvan, M.S. 2014. Sensor-based real-time resource model reconciliation for improved mine production control: a conceptual framework. Proceedings of the SMP Symposium on Orebody Modelling and Strategic Mine Planning: Integrated Mineral Investment and Supply Chain Optimisation, Perth, Australia, 24-26 November 2014. Australasian Institute of Minng and Metallurgy, Melbourne.

Chiles, J.P. and Delfiner, P. 2012. Geostatistics: Modeling Spatial Uncertainty. 2nd edn. Wiley, Hoboken, NJ.

Dowd, P.A. and Dare-Bryan, P. 2005. Planning, designing and optimising production using geostatistical simulation. Proceedings of the
International Symposium on Orebody Modelling and Strategic Mine

Planning: Uncertainty and Risk Management, Perth. Australasian

Institute of Minng and Metallurgy, Melbourne. pp. 321-338.

Fioroni, M.M., Franzese, L.A.G., Zanin, C.E., Fúria, J., De Toledo Perfetti, L., LEONARDo, D., and DA SILVA, N.L. 2007. Simulation of continuous behavior using discrete tools: ore conveyor transport. Proceedings of the 39th Conference on Winter simulation: 40 years! The Best is Yet to Come. IEEE Press. pp. 1655-1662.

IsAAKS, E.H. and SRIVASTAVA, R.M. 1989. An Introduction to Applied Geostatistics. Oxford University Press.

Kelton, W.D. and Law, A.M. 2000. Simulation Modeling and Analysis. McGraw Hill, Boston, MA.

Michalakopoulos, T.N., Arvaniti, S., and Panagiotou, G.N. 2005. Simulation of a continuous lignite excavation system. Proceedings of the 14th International Symposium on Mine Planning and Equipment Selection (MPES 2005), Banff, Alberta, 31 October-3 November. Singhal, R., Fytas, K., and Chiwetelu, C. (eds). Reading Matrix.

Michalakopoulos, T.N., Roumpos, C.P., Galetakis, M.J., and Panagiotou, G.N. 2015. Discrete-event simulation of continuous mining systems in multilayer lignite deposits. Proceedings of the 12th International Symposium on Continuous Surface Mining, ISCSM Aachen 2014 . Springer. pp. 225-239.

PANAGIOTou, G.N. 1983. Computer simulation of the mining operations in opencast lignite mines operating BWEs, conveyors and stackers. Proceedings of the First Conference on Use of Computers in the Coal Industry, Morgantown, WV, 1-3 August 1983. SME, New York. pp. $150-157$.

Rockwell Automation Technologies Inc. 2012. Arena (Version 14.50.00000 CPR 9 SR 2).

Salama, A., Nehring, M., and Greberg, J. 2014. Operating value optimisation using simulation and mixed integer programming. International Journal of Mining, Reclamation and Environment, vol. 28, no. 1. pp. 25-46.

SCHIFFER, H.-W. and MAASSEN, U. 2013. DEBRIV: Braunkohle in Deutschland 2013 - Profil eines Industriezweiges. www.braunkohle.de [Accessed 15 November. 2014].

ShISHVAn, M.S. and BenNDoRf, J. 2014. Performance optimization of complex continuous mining system using stochastic simulation. Proceedings of Engineering Optimization 2014, the 4th Internationa Conference on Engineering Optimization, Lisbon, Portugal, 8-11 September 2014. CRC Press, Boca Raton, FL. pp. 273-278. 